\title{
Global existence of solutions for a free boundary problem modeling the growth of necrotic tumors
}

\author{
SHANGBIN CUI ${ }^{\dagger}$ \\ Institute of Mathematics, Sun Yat-Sen University, Guangzhou, \\ Guangdong 510275, People's Republic of China
}

[Received 9 January 2004 and in revised form 22 November 2004]

\begin{abstract}
In this paper we study a free boundary problem for a reaction-diffusion equation modeling the growth of necrotic tumors. We first reduce this problem into an equivalent initial boundary value problem for a nonlinear parabolic equation on a fixed domain. This parabolic equation is strongly singular in the sense that not only it contains a discontinuous nonlinear function of the unknown function, but also all its coefficients are discontinuous nonlinear functionals of the unknown function. We use the approximation method and the Schauder fixed point theorem combined with $L^{p}$ estimates to prove the existence of a global solution.
\end{abstract}

2000 Mathematics Subject Classification: 35K35, 35Q80, 35R05.

Keywords: Free boundary problem; tumor growth; global solution; existence.

\section{Introduction}

Recent development on mathematical modeling of tumor growth has introduced many new interesting free boundary problems for partial differential equations [1, 4, 6, 15, -21]. These free boundary problems are very diversified: Some of them involve only reaction-diffusion equations but possibly with more than one free boundaries [1, 4, 6, 15, 16, 19], others involve not only reactiondiffusion equations but also first order hyperbolic equations [17, 18, 20, 21]. Rigorous mathematical analysis of such free boundary problems has drawn great interest, and many interesting results have been established [2, 3, 7,-14].

In this paper we study the following free boundary problem modeling the growth of spherically symmetric necrotic tumors:

$$
\begin{gathered}
\sigma_{t}(|x|, t)=D \triangle \sigma(|x|, t)-\lambda N_{L} \sigma(|x|, t) H\left(\sigma(|x|, t)-\sigma_{D}\right), \quad|x|<R(t), t \geqslant 0, \\
\sigma(R(t), t)=\sigma_{S}, \quad t \geqslant 0, \\
\frac{\mathrm{d}}{\mathrm{d} t}\left(\frac{4}{3} \pi R^{3}(t)\right)=\mu N_{L} \int_{\sigma(|x|, t)>\sigma_{D}}\left(\sigma(|x|, t)-\sigma_{D}\right) \mathrm{d} x \\
-v N_{D} \int_{\sigma(|x|, t)>\sigma_{D}} \mathrm{~d} x-v N \int_{\sigma(|x|, t) \leqslant \sigma_{D}} \mathrm{~d} x, \\
\sigma(|x|, 0)=\sigma_{0}(|x|), \quad|x| \leqslant R_{0}, \\
R(0)=R_{0} .
\end{gathered}
$$

${ }^{\dagger}$ E-mail: cuisb3@yahoo.com.cn 
Here $\sigma(|x|, t)$ is the concentration of nutrient which is regarded as a one-species chemical material, $R(t)$ is the radius of the tumor at time $t, D$ is the diffusion coefficient, $\Delta$ is the Laplacian, $\lambda$ is the consumption rate coefficient of nutrient for live tumor cells, $N_{L}$ is the density of live tumor cells, $H$ is the Heaviside function: $H(s)=1$ for $s>0$ and $H(s)=0$ for $s \leqslant 0, \sigma_{S}$ is a positive constant representing the constant supply of nutrient that the tumor receives from its surface, $\mu$ is the mitosis rate coefficient of live tumor cells when the nutrient concentration is at the level $\sigma-\sigma_{D}, \sigma_{D}$ is a positive constant representing the threshold value such that in the region where $\sigma \leqslant \sigma_{D}$ all tumor cells are dead and only in the region where $\sigma>\sigma_{D}$ tumor cells can be alive, $N_{D}$ is the density of dead cells, $v$ is the dissolution rate of dead cells, $N$ is the density of all (live and dead) tumor cells, and $\sigma_{0}(|x|)$ and $R_{0}$ are the initial data of $\sigma$ and $R$, respectively. We assume that the tumor is well-packed with cells such that the density of cells (regardless of whether they are alive or dead) is everywhere equal, so that

$$
N_{D}+N_{L}=N
$$

and the chemicals dissolved from the dead cells diffuse into the solution in which the tumor is cultivated. The constant $N_{D}$ reflects the constant cell death rate due to apoptosis. It is natural to assume that

$$
0 \leqslant \sigma_{0}(|x|) \leqslant \sigma_{S} \quad \text { for }|x| \leqslant R_{0}, \quad \text { and } \sigma_{0}\left(R_{0}\right)=\sigma_{S} .
$$

The above model is in essence a combination of the two tumor growth models proposed by Byrne and Chaplain: in [4] for non-necrotic tumors and in [5] for necrotic tumors, but here the effects of inhibitors and vascular networks have been neglected. Indeed, in the case that $\sigma(|x|, t)>\sigma_{D}$ for all $|x| \leqslant R(t)$ and all $t \geqslant 0$, we have $H\left(\sigma_{D}(|x|, t)-\sigma_{D}\right) \equiv 1$, so that [1.1 - 1.5 reduces to the model of Byrne and Chaplain [4] in the inhibitor-free and avascular situation, and in the opposite case it is a reformulation of the model of Byrne and Chaplain [6], also in the inhibitor-free and avascular situation.

In the general case the problem $1.1-(1.5)$ contains two free boundaries: the outer tumor surface $|x|=R(t)$ and the inner interface between the necrotic core $\left\{x: \sigma(|x|, t) \leqslant \sigma_{D}\right\}$ and the live shell $\left\{x: \sigma(|x|, t)>\sigma_{D}\right\}$, and the two free boundaries are of two different types: the tumor surface $|x|=R(t)$ is of Stefan (evolutionary) type while the inner interface is of obstacle (stationary or non-evolutionary) type. It turns out that rigorous mathematical treatment of this problem is hard.

In [9] the author and A. Friedman made a partial analysis of the problem (1.1)-(1.5). It was proved that under certain sufficient conditions this problem has a unique stationary solution and, when the initial data belong to a small neighborhood of the stationary solution and satisfy some other very rigid conditions, it has a unique global classical solution which tends to the stationary solution as $t \rightarrow \infty$.

In this work we shall prove that the problem $1.1-(1.5)$ has a solution for all $t \geqslant 0$ under the conditions (1.7) and

$$
\sigma_{0} \in W^{2, \infty}\left(0, R_{0}\right) \quad \text { and } \quad \sigma_{0}^{\prime}(0)=0 .
$$

Here $W^{2, \infty}\left(0, R_{0}\right)=\left\{\varphi \in L^{\infty}\left(0, R_{0}\right): \varphi^{\prime}, \varphi^{\prime \prime} \in L^{\infty}\left(0, R_{0}\right)\right\}$. Clearly, this result greatly improves the existence result of [9]. The idea we use to obtain this result is different from that of [9]. Indeed, the argument of [9] is as follows: For a given $T>0$, we first assume $R=R(t)(0 \leqslant t \leqslant T)$ to be given, and solve the problem (1.1), (1.2) and (1.4) to find $\sigma=\sigma(|x|, t)$. Then we substitute this $\sigma(|x|, t)$ into $(1.3)$, and solve (1.3) and (1.5) to get a new $R=R(t)$. By using the Banach fixed point theorem we then get a solution $(\sigma, R)$ for small $T$. For that argument we need a delicate analysis of regularity of the solution of the problem (1.1), (1.2) and (1.4) (for given $R=R(t)$ ), which is hard 
and quite restricted (i.e., the initial data have to satisfy some very rigid conditions). The method we use in the present work is much simpler, and can be explained as follows: Let $r=|x|$ and introduce a new variable $z=r / R(t)$. Then the time-dependent unknown interval $[0, R(t)]$ is transformed into the fixed interval $[0,1]$, and the free boundary problem $(1.1)-(1.5)$ is transformed into an initial boundary value problem on the fixed domain $[0,1]$ for the new unknowns $v(z, t)=\sigma(z R(t), t)$ and $R(t)$. In the transformed problem $R(t)$ can be found in terms of $v$ (as one can observe from the equation (1.3)), so that the equation for $v(z, t)$ can be decoupled. The decoupled equation for $v(z, t)$ is a nonlinear parabolic equation which is strongly singular in the sense that, apart from the discontinuous nonlinear term $v H\left(v-\sigma_{D}\right)$ inherited from the equation (1.1), its coefficients are discontinuous nonlinear functionals in $(v, t)$. We shall use the approximation method and the Schauder fixed point theorem combined with $L^{p}$ estimates to solve this equation.

As we pointed out earlier, the effect of inhibitors in the present model is neglected. This is merely for the purpose of simplicity of the statement. The argument presented in this paper can be easily modified to get similar results for tumor models with the effect of inhibitors taken into account.

In the following section we shall show how to reduce the problem $\sqrt{1.1}-(1.5)$ to a scalar parabolic equation involving discontinuous terms. In $\S 3$ we shall approximate this equation with a smooth equation, and use the Schauder fixed point theorem and $L^{p}$ estimates to prove that the approximation problem has a unique global solution. In $\S 4$ we use $L^{p}$ estimates and weak convergence to prove that the problem (1.1) $-(1.5)$ has a solution under conditions $(1.7)$ and $(1.8)$.

\section{Reduction of the problem}

For simplicity of notation, we rescale the space variable so that

$$
\frac{\lambda N_{L}}{D}=1 .
$$

Then the problem 1.1 -1.5 can be reformulated as follows:

$$
\begin{gathered}
c \sigma_{t}=\triangle_{r} \sigma(r, t)-\sigma(r, t) H\left(\sigma(r, t)-\sigma_{D}\right), \quad 0<r<R(t), \quad t \geqslant 0, \\
\dot{R}(t)=\frac{\bar{\mu}}{R^{2}(t)}\left\{\int_{\sigma(r, t)>\sigma_{D}}(\sigma(r, t)-\tilde{\sigma}) r^{2} \mathrm{~d} r-\bar{v} \int_{\sigma(r, t) \leqslant \sigma_{D}} r^{2} \mathrm{~d} r\right\}, \quad t>0, \\
\sigma(r, t)=\sigma_{0}(r), \quad 0 \leqslant r \leqslant R_{0}, \\
R(0)=R_{0},
\end{gathered}
$$

where

$$
\triangle_{r} \sigma=\frac{1}{r^{2}} \frac{\partial}{\partial r}\left(r^{2} \frac{\partial \sigma}{\partial r}\right)
$$

and

$$
c=\frac{1}{D}, \quad \bar{\mu}=\mu N_{L}, \quad \tilde{\sigma}=\sigma_{D}+\frac{\nu N_{D}}{\mu N_{L}}, \quad \bar{v}=\frac{\nu N}{\mu N_{L}} .
$$

We shall always assume that the conditions (1.7) and (1.8) are satisfied. 
It is clear that if $\sigma_{S} \leqslant \sigma_{D}$ then $\sigma(|x|, t) \leqslant \sigma_{D}$ for all $0 \leqslant r \leqslant R(t)$ and $t \geqslant 0$, so that $R(t)=$ $R_{0} e^{-v N t / 3}$, and $\sigma(r, t)=\sigma_{S}$. Biologically, this means that no place of the tumor has enough nutrient to sustain cells alive, so that the tumor contains only dead cells which are decreasing in amount due to dissolution, and finally the tumor will disappear. This trivial situation is not interesting. Later on we always assume that

$$
\sigma_{S}>\sigma_{D}
$$

and will not repeat this assumption. Biologically, 2.8) implies that at least in the near-surface region there is sufficient nutrient to sustain tumor cells alive. Thus we can expect that the tumor will always exist.

We introduce a transformation of variables $(r, t, \sigma, R) \mapsto(z, t, v, R)$ as follows:

$$
z=\frac{r}{R(t)}, \quad t=t, \quad v(z, t)=\sigma(z R(t), t), \quad R(t)=R(t)
$$

One can easily verify that, under this transformation of variables, the problem 2.1 -2.6 is transformed into the following problem:

$$
\begin{gathered}
c v_{t}=\frac{1}{R^{2}(t)} \triangle_{z} v+c \frac{\dot{R}(t)}{R(t)} \cdot z v_{z}-v H\left(v-\sigma_{D}\right), \quad 0<z<1, t>0, \\
v_{z}(0, t)=0, \quad v(1, t)=\sigma_{S}, \quad t \geqslant 0, \\
\dot{R}(t)=\bar{\mu} R(t)\left\{\int_{v(z, t)>\sigma_{D}}(v(z, t)-\tilde{\sigma}) z^{2} \mathrm{~d} z-\bar{v} \int_{v(z, t) \leqslant \sigma_{D}} z^{2} \mathrm{~d} z\right\}, \quad t>0, \\
v(z, 0)=v_{0}(z) \equiv \sigma_{0}\left(z R_{0}\right), \quad 0 \leqslant z \leqslant 1, \\
R(0)=R_{0} .
\end{gathered}
$$

For a given $T>0$, we denote by $X_{T}$ the function space $C([0,1] \times[0, T])$ endowed with the maximum norm $\|v\|=\max _{[0,1] \times[0, T]}|v(z, t)|$, and denote by $F$ the functional on $X_{T} \times[0, T]$ defined by

$$
F(v, t)=\bar{\mu}\left\{\int_{v(z, t)>\sigma_{D}}(v(z, t)-\tilde{\sigma}) z^{2} \mathrm{~d} z-\bar{v} \int_{v(z, t) \leqslant \sigma_{D}} z^{2} \mathrm{~d} z\right\} .
$$

Then the equation 2.12 can be rewritten as

$$
\dot{R}(t)=R(t) F(v, t), \quad t>0 .
$$

It follows that $R(t)$ can be expressed in terms of $v$ by

$$
R(t)=R_{0} \exp \left(\int_{0}^{t} F(v, \tau) \mathrm{d} \tau\right), \quad t \geqslant 0 .
$$

Substituting this expression and 2.16) into the equation 2.10), we get a scalar equation for $v$ :

$$
\begin{aligned}
c v_{t}= & R_{0}^{-2} \exp \left(-2 \int_{0}^{t} F(v, \tau) \mathrm{d} \tau\right) \triangle_{z} v \\
& +c F(v, t) z v_{z}-v H\left(v-\sigma_{D}\right), \quad 0<z<1, \quad t>0 .
\end{aligned}
$$

Hence, the problem 2.1)-2.6 reduces to the problem 2.11, 2.13, 2.15) and 2.18. 
The functional $F(v, t)$ is not continuous in $v$. Indeed, if we denote by $w$ the function in $X_{T}$ :

$$
w(z, t)= \begin{cases}\sigma_{D} & \text { for } 0 \leqslant z \leqslant a, 0 \leqslant t \leqslant T, \\ \sigma_{D}+\frac{z-a}{1-a}\left(\sigma_{S}-\sigma_{D}\right) & \text { for } a<z \leqslant 1,0 \leqslant t \leqslant T,\end{cases}
$$

where $0<a<1$, and set $v_{\varepsilon}=w+\varepsilon\left(\sigma_{s}-w\right)(\varepsilon>0)$, then clearly

$$
\lim _{\varepsilon \rightarrow 0} v_{\varepsilon}=w, \quad \text { but } \quad \lim _{\varepsilon \rightarrow 0} F\left(v_{\varepsilon}, t\right)=F(w, t)+\frac{1}{3} \bar{\mu} a^{3}\left(\sigma_{D}-\tilde{\sigma}+\bar{v}\right) \neq F(w, t) .
$$

Recalling $v_{0}(z)=\sigma_{0}\left(z R_{0}\right)$ (see $(2.13)$, we see, by the conditions $(1.7)$ and $(1.8)$, that

$$
v_{0} \in W^{2, \infty}(0,1), \quad 0 \leqslant v_{0}(z) \leqslant \sigma_{S} \quad \text { for } 0 \leqslant z \leqslant 1, \quad v_{0}^{\prime}(0)=0, \quad v_{0}(1)=\sigma_{S} .
$$

\section{The approximation problem}

Since the equation 2.18 contains discontinuous terms, it is singular. In this section we shall approximate it with smooth equations.

First, we note that the functional $F$ can be rewritten as follows:

$$
F(v, t)=\bar{\mu}\left\{\int_{0}^{1}(v(z, t)-\tilde{\sigma}) H\left(v(z, t)-\sigma_{D}\right) z^{2} \mathrm{~d} z-\bar{v} \int_{0}^{1}\left(1-H\left(v(z, t)-\sigma_{D}\right)\right) z^{2} \mathrm{~d} z\right\} .
$$

For each sufficiently small $\varepsilon>0$, we denote by $H_{\varepsilon}$ the function on $\mathbb{R}$ defined by

$$
H_{\varepsilon}(s)= \begin{cases}1 & \text { for } s \geqslant \varepsilon \\ s / \varepsilon & \text { for } 0<s<\varepsilon \\ 0 & \text { for } s \leqslant 0\end{cases}
$$

Clearly, $H_{\varepsilon}$ is a Lipschitz continuous function:

$$
\left|H_{\varepsilon}(s)-H_{\varepsilon}(t)\right| \leqslant \varepsilon^{-1}|s-t|, \quad \forall s, t \in \mathbb{R} .
$$

We define

$$
\begin{aligned}
F_{\varepsilon}(v, t)=\bar{\mu}\left\{\int_{0}^{1}(v(z, t)-\tilde{\sigma}) H_{\varepsilon}\left(v(z, t)-\sigma_{D}\right) z^{2} \mathrm{~d} z\right. & \\
& \left.-\bar{v} \int_{0}^{1}\left(1-H_{\varepsilon}\left(v(z, t)-\sigma_{D}\right)\right) z^{2} \mathrm{~d} z\right\},
\end{aligned}
$$

and consider the problem

$$
\begin{gathered}
c v_{t}=R_{0}^{-2} \exp \left(-2 \int_{0}^{t} F_{\varepsilon}(v, \tau) d \tau\right) \triangle_{z} v \\
+c F_{\varepsilon}(v, t) z v_{z}-v H_{\varepsilon}\left(v-\sigma_{D}\right) \quad \text { for } 0<z<1, t>0, \\
v_{z}(0, t)=0, \quad v(1, t)=\sigma_{S} \quad \text { for } t \geqslant 0, \\
v(z, 0)=v_{0}(z) \quad \text { for } 0 \leqslant z \leqslant 1 .
\end{gathered}
$$


In what follows we use the notation:

$$
\begin{gathered}
B_{1}(0)=\left\{y \in \mathbb{R}^{3}:|y|<1\right\}, \quad Q_{T}=B_{1}(0) \times(0, T), \\
W_{p}^{2,1}\left(Q_{T}\right)=\left\{v \in L^{p}\left(Q_{T}\right): \nabla v, \nabla^{2} v, v_{t} \in L^{p}\left(Q_{T}\right)\right\} \quad(1 \leqslant p \leqslant \infty) .
\end{gathered}
$$

Here and hereafter $\nabla v$ and $\nabla^{2} v$ respectively represent the divergence vector and the Hessian matrix of $v$ in space variables.

LEMmA 3.1 Under the condition (2.19), for any $T>0$ the problem $3.3-(3.5)$ has a unique solution $v=v_{\varepsilon}$ on $[0,1] \times[0, T]$, with the following properties:

(i) The function $v_{\varepsilon}(|y|, t)$ belongs to $W_{p}^{2,1}\left(Q_{T}\right)$ for any $1<p<\infty$, and $v_{\varepsilon}(z, t)$ satisfies the equation for a.e. $(z, t) \in[0,1] \times[0, T]$. Moreover, for any $\varepsilon>0$,

$$
\left\|v_{\mathcal{E}}(|y|, t)\right\|_{W_{p}^{2,1}\left(Q_{T}\right)} \leqslant C\left(T, p,\left\|v_{0}\right\|_{W^{2, \infty}(0,1)}\right),
$$

where $C\left(T, p,\left\|v_{0}\right\|_{W^{2, \infty}(0,1)}\right)$ is a constant independent of $\varepsilon$.

(ii) For every $\varepsilon>0$,

$$
0 \leqslant v_{\varepsilon}(z, t) \leqslant \sigma_{S} \quad \text { for } 0 \leqslant z \leqslant 1,0 \leqslant t \leqslant T .
$$

If in addition $v_{0}^{\prime}(z) \geqslant 0$ then also $v_{\varepsilon z}(z, t) \geqslant 0$.

Proof. Let $X_{T}$ be as before, i.e., $X_{T}=C([0,1] \times[0, T])$ endowed with the maximum norm $\|v\|=\max _{[0,1] \times[0, T]}|v(z, t)|$. We define a mapping $S: X_{T} \rightarrow X_{T}$ as follows: For any $v \in X_{T}$, let $\tilde{v}=\tilde{v}(y, t)\left((y, t) \in \bar{Q}_{T}\right)$ be the solution of the problem

$$
\begin{gathered}
c \tilde{v}_{t}=R_{0}^{-2} \exp \left(-2 \int_{0}^{t} F_{\varepsilon}(v, \tau) \mathrm{d} \tau\right) \triangle \tilde{v} \\
+c F_{\varepsilon}(v, t)(y \cdot \nabla \tilde{v})-H_{\varepsilon}\left(v(|y|, t)-\sigma_{D}\right) \tilde{v} \quad \text { on } Q_{T} \\
\tilde{v}(y, t)=\sigma_{S} \quad \text { for }|y|=1, t \geqslant 0 \\
\tilde{v}(y, 0)=v_{0}(|y|) \quad \text { for }|y| \leqslant 1
\end{gathered}
$$

$\tilde{v}$ is well defined. Indeed, it is clear that all coefficients in the equation (3.8) are bounded continuous functions, and the coefficient of $\Delta \tilde{v}$ has a positive lower bound. Hence, using the $L^{p}$ theory for linear parabolic equations we see that the above problem has a unique solution $\tilde{v}$ defined on $Q_{T}$, such that for any $1<p<\infty, \tilde{v} \in W_{p}^{2,1}\left(Q_{T}\right)$, i.e.,

$$
\tilde{v}, \nabla \tilde{v}, \nabla^{2} \tilde{v}, \tilde{v}_{t} \in L^{p}\left(Q_{T}\right)
$$

We take in particular $p>5 / 2$. Then by the embedding $W_{p}^{2,1}\left(Q_{T}\right) \hookrightarrow C\left(\bar{Q}_{T}\right)(p>5 / 2)$, we see that $\tilde{v} \in C\left(\bar{Q}_{T}\right)$. Moreover, since the boundary and initial data are spherically symmetric, by uniqueness we infer that $\tilde{v}(y, t)$ is also spherically symmetric in $y$, so that $\tilde{v}(y, t)=\tilde{v}(|y|, t)$. In this way we get a function $\tilde{v}=\tilde{v}(z, t)$ defined for $(z, t) \in[0,1] \times[0, T]$, belonging to $X_{T}$. We define $S(v)=\tilde{v}$.

Next we prove that for every bounded subset $E \subset X_{T}, S(E)$ is precompact in $X_{T}$. In fact, it is clear that

$$
\left|F_{\varepsilon}(v, t)\right| \leqslant C(\|v\|) \quad \text { for all } v \in X_{T}, t \in[0, T]
$$


where $C(\|v\|)$ represents a constant depending only on $\|v\|=\max _{[0,1] \times[0, T]}|v(z, t)|$ (independent of $\varepsilon$ and $T$ ). This further implies that

$$
e^{-2 T C(\|v\|)} \leqslant \exp \left(-2 \int_{0}^{t} F_{\varepsilon}(v, \tau) \mathrm{d} \tau\right) \leqslant e^{2 T C(\|v\|)} \quad \text { for } v \in X_{T}, t \in[0, T],
$$

and

$$
\begin{aligned}
\left|\exp \left(-2 \int_{0}^{t} F_{\varepsilon}(v, \tau) \mathrm{d} \tau\right)-\exp \left(-2 \int_{0}^{t^{\prime}} F_{\varepsilon}(v, \tau) \mathrm{d} \tau\right)\right| & \\
\leqslant C(\|v\|, T)\left|t-t^{\prime}\right| & \text { for } v \in X_{T}, t \in[0, T] .
\end{aligned}
$$

Since also

$$
0 \leqslant H_{\varepsilon}\left(v(|y|, t)-\sigma_{D}\right) \leqslant 1 \quad \text { for } y \in \bar{B}_{1}(0), t \in[0, T],
$$

by standard $L^{p}$ estimates for linear parabolic equations we conclude that for any $1<p<\infty$ there exists a constant $C(\|v\|, T, p)$ such that

$$
\|\tilde{v}(|y|, t)\|_{W_{p}^{2,1}\left(Q_{T}\right)} \leqslant C(\|v\|, T, p)\left(\sigma_{S}+\left\|v_{0}\right\|_{W^{2, \infty}(0,1)}\right) .
$$

By this estimate and the compact embedding of $W_{p}^{2,1}\left(Q_{T}\right)$ into $C\left(\bar{Q}_{T}\right)$ when $p>5 / 2$, we conclude that if $E \subset X_{T}$ is bounded in $X_{T}$ then $S(E)$ is precompact in $X_{T}$. Further, by the maximum principle it is clear that

$$
0 \leqslant \tilde{v}(z, t) \leqslant \sigma_{S} \text { for } 0 \leqslant z \leqslant 1,0 \leqslant t \leqslant T .
$$

We now prove that $S$ is continuous. Let $v_{1}, v_{2} \in X_{T}$ and define $\tilde{v}_{1}=S\left(v_{1}\right), \tilde{v}_{2}=S\left(v_{2}\right)$, $w=\tilde{v}_{1}-\tilde{v}_{2}$. Then $w=w(|y|, t)$ satisfies

$$
\begin{gathered}
c w_{t}=R_{0}^{-2} \exp \left(-2 \int_{0}^{t} F_{\varepsilon}\left(v_{1}, \tau\right) \mathrm{d} \tau\right) \Delta w+c F_{\varepsilon}\left(v_{1}, t\right)(y \cdot \nabla w) \\
-H_{\varepsilon}\left(v_{1}(|y|, t)-\sigma_{D}\right) w+f(y, t) \quad \text { for }|y|<1,0<t \leqslant T, \\
w(y, t)=0 \quad \text { for }|y|=1,0 \leqslant t \leqslant T, \\
w(y, 0)=0 \quad \text { for }|y| \leqslant 1,
\end{gathered}
$$

where

$$
\begin{aligned}
f(y, t)= & R_{0}^{-2}\left\{\exp \left(-2 \int_{0}^{t} F_{\varepsilon}\left(v_{1}, \tau\right) \mathrm{d} \tau\right)-\exp \left(-2 \int_{0}^{t} F_{\varepsilon}\left(v_{2}, \tau\right) \mathrm{d} \tau\right)\right\} \Delta \tilde{v}_{2} \\
& +c\left(F_{\varepsilon}\left(v_{1}, t\right)-F_{\varepsilon}\left(v_{2}, t\right)\right)\left(y \cdot \nabla \tilde{v}_{2}\right) \\
& -\left(H_{\varepsilon}\left(v_{1}(|y|, t)-\sigma_{D}\right)-H_{\varepsilon}\left(v_{2}(|y|, t)-\sigma_{D}\right)\right) \tilde{v}_{2} .
\end{aligned}
$$

By the $L^{p}$ estimate and (3.11)-(3.14) we have, for any $1<p<\infty$,

$$
\|w(y, t)\|_{W_{p}^{2,1}\left(Q_{T}\right)} \leqslant C\left(\left\|v_{1}\right\|, T, p\right)\|f\|_{L^{p}\left(Q_{T}\right)} .
$$

Using Lipschitz continuity of $H_{\varepsilon}$ and 3.15 one can easily verify that

$$
\|f\|_{L^{p}\left(Q_{T}\right)} \leqslant C\left(\varepsilon,\left\|v_{2}\right\|, T, p\right)\left\|v_{1}-v_{2}\right\| .
$$


Substituting this estimate into 3.21 and using the embedding inequality for $W_{p}^{2,1}\left(Q_{T}\right) \hookrightarrow C\left(Q_{T}\right)$ when $p>5 / 2$ we get

$$
\left\|\tilde{v}_{2}-\tilde{v}_{1}\right\| \leqslant C(T, p)\|w\|_{W_{p}^{2,1}\left(Q_{T}\right)} \leqslant C\left(\varepsilon,\left\|v_{1}\right\|,\left\|v_{2}\right\|, T, p\right)\left\|v_{1}-v_{2}\right\| .
$$

Hence, $S$ is continuous.

Now let $E=\left\{v \in X_{T}: 0 \leqslant v(z, t) \leqslant \sigma_{S}\right.$ for $\left.0 \leqslant z \leqslant 1,0 \leqslant t \leqslant T\right\}$. Then by (3.16), $S$ maps $E$ into itself. It follows by the Schauder fixed point theorem that $S$ has a fixed point in $E$. Since it is clear that a fixed point of $S$ is a solution of the problem (3.3)-(3.5), we have thus proved that this problem has a solution. Moreover, since any solution of $3.3-3.5$ is a fixed point of $S$, by 3.15 and (3.16), any solution $v=v_{\varepsilon}$ of (3.3) -3.5 satisfies

$$
\left\|v_{\varepsilon}(|y|, t)\right\|_{W_{p}^{2,1}\left(Q_{T}\right)} \leqslant C(T, p)\left(\sigma_{S}+\left\|v_{0}\right\|_{W^{2, \infty}(0,1)}\right)
$$

for any $1<p<\infty$. We note that the constant $C(T, p)$ is independent of $\varepsilon$. Hence (3.6) holds.

Next we prove the uniqueness. Let $v_{1}$ and $v_{2}$ be two solutions of the problem 3.3 -3.5 and define $w=v_{1}-v_{2}$. Then $w=w(|y|, t)$ satisfies

$$
\begin{gathered}
c w_{t}=R_{0}^{-2} \exp \left(-2 \int_{0}^{t} F_{\varepsilon}\left(v_{1}, \tau\right) \mathrm{d} \tau\right) \Delta w+c F_{\varepsilon}\left(v_{1}, t\right)(y \cdot \nabla w) \\
-\left\{H_{\varepsilon}\left(v_{1}(|y|, t)-\sigma_{D}\right) v_{1}-H_{\varepsilon}\left(v_{2}(|y|, t)-\sigma_{D}\right) v_{2}\right\}+g(y, t), \quad|y|<1, t>0, \\
w(y, t)=0, \quad|y|=1, t \geqslant 0, \\
w(y, 0)=0, \quad|y| \leqslant 1,
\end{gathered}
$$

where

$$
\begin{aligned}
g(y, t)= & \left\{\exp \left(-2 \int_{0}^{t} F_{\varepsilon}\left(v_{1}, \tau\right) \mathrm{d} \tau\right)-\exp \left(-2 \int_{0}^{t} F_{\varepsilon}\left(v_{2}, \tau\right) \mathrm{d} \tau\right)\right\} \Delta v_{2}(|y|, t) \\
& +c\left(F_{\varepsilon}\left(v_{1}, t\right)-F_{\varepsilon}\left(v_{2}, t\right)\right)\left(y \cdot \nabla v_{2}(|y|, t)\right)
\end{aligned}
$$

Multiplying (3.23) with $w$ and integrating in $y$ over $B_{1}(0)$ we get

$$
\begin{aligned}
\frac{c}{2} \frac{\mathrm{d}}{\mathrm{d} t} \int_{B_{1}(0)}|w(|y|, t)|^{2} \mathrm{~d} y \leqslant & -R_{0}^{-2} \exp \left(-2 \int_{0}^{t} F_{\varepsilon}\left(v_{1}, \tau\right) \mathrm{d} \tau\right) \int_{B_{1}(0)}|\nabla w(|y|, t)|^{2} \mathrm{~d} y \\
& +c F_{\varepsilon}\left(v_{1}, t\right) \int_{B_{1}(0)}(y \cdot \nabla w(|y|, t)) w(|y|, t) \mathrm{d} y \\
& +\int_{B_{1}(0)} g(y, t) w(|y|, t) \mathrm{d} y
\end{aligned}
$$

Since

$$
\int_{B_{1}(0)}(y \cdot \nabla w(|y|, t)) w(|y|, t) \mathrm{d} y=-\frac{3}{2} \int_{B_{1}(0)}|w(|y|, t)|^{2} \mathrm{~d} y
$$


integrating (3.27) in $t$ over $[0, t]$ for any $t \in(0, T)$ and using 3.11 and (3.12) we get

$$
\begin{aligned}
\int_{B_{1}(0)}|w(|y|, t)|^{2} \mathrm{~d} y \leqslant & -C_{1}(T) \int_{0}^{t} \int_{B_{1}(0)}|\nabla w(|y|, \tau)|^{2} \mathrm{~d} y \mathrm{~d} \tau \\
& +C_{2}(T) \int_{0}^{t} \int_{B_{1}(0)}|w(|y|, \tau)|^{2} \mathrm{~d} y \mathrm{~d} \tau \\
& +\int_{0}^{t} \int_{B_{1}(0)} g(y, \tau) w(|y|, \tau) \mathrm{d} y \mathrm{~d} \tau .
\end{aligned}
$$

By Lipschitz continuity of $H_{\varepsilon}$ and boundedness of $v_{1}$ and $v_{2}$, it is clear that

$$
\left|F_{\varepsilon}\left(v_{1}, t\right)-F_{\varepsilon}\left(v_{2}, t\right)\right| \leqslant C(\varepsilon) \int_{B_{1}(0)}\left|v_{1}(|y|, t)-v_{2}(|y|, t)\right| \mathrm{d} y=C(\varepsilon) \int_{B_{1}(0)}|w(|y|, t)| \mathrm{d} y .
$$

Hence

$$
\begin{aligned}
& \int_{0}^{t} \int_{B_{1}(0)} g(y, \tau) w(|y|, \tau) \mathrm{d} y \mathrm{~d} \tau \\
& \leqslant C(\varepsilon, T) \int_{0}^{t}\left\{\left|F_{\varepsilon}\left(v_{1}, \tau\right)-F_{\varepsilon}\left(v_{2}, \tau\right)\right| \int_{B_{1}(0)}\left|\nabla v_{2}(|y|, \tau) \cdot \nabla w(|y|, \tau)\right| \mathrm{d} y\right\} \mathrm{d} \tau \\
& \quad+c \int_{0}^{t}\left\{\left|F_{\varepsilon}\left(v_{1}, \tau\right)-F_{\varepsilon}\left(v_{2}, \tau\right)\right| \int_{B_{1}(0)}\left|\nabla v_{2}(|y|, \tau)\right||w(|y|, \tau)| \mathrm{d} y\right\} \mathrm{d} \tau \\
& \leqslant C(\varepsilon, T) \int_{0}^{t}\left\{\left(\int_{B_{1}(0)}|w(|y|, \tau)| \mathrm{d} y\right)\left(\int_{B_{1}(0)}\left|\nabla v_{2}(|y|, \tau)\right|(|\nabla w(|y|, \tau)|+|w(|y|, \tau)|) \mathrm{d} y\right)\right\} \mathrm{d} \tau \\
& \leqslant C(\varepsilon, T) \max _{\left(y, t^{\prime}\right) \in Q_{T}}\left|\nabla v_{2}\left(|y|, t^{\prime}\right)\right|\left\{\delta \int_{0}^{t} \int_{B_{1}(0)}|\nabla w(|y|, \tau)|^{2} \mathrm{~d} y \mathrm{~d} \tau\right. \\
& \left.\quad+C(\delta) \int_{0}^{t} \int_{B_{1}(0)}|w(|y|, \tau)|^{2} \mathrm{~d} y \mathrm{~d} \tau\right\}
\end{aligned}
$$

where $\delta$ is a sufficiently small positive number and $C(\delta)$ is a constant depending on $\delta$. Since for $p>5$ we have

$$
\max _{Q_{T}}\left|\nabla v_{2}(|y|, t)\right| \leqslant C(T, p)\left\|v_{2}\right\|_{W_{p}^{2,1}\left(Q_{T}\right)},
$$

using 3.22 we get

$$
\max _{Q_{T}}\left|\nabla v_{2}(|y|, t)\right| \leqslant C(T) .
$$

Hence, by taking $\delta$ sufficiently small we have

$$
\begin{aligned}
\int_{0}^{t} \int_{B_{1}(0)} g(y, \tau) w(|y|, \tau) \mathrm{d} y \mathrm{~d} \tau \leqslant & \frac{1}{2} C_{1}(T) \int_{0}^{t} \int_{B_{1}(0)}|\nabla w(|y|, \tau)|^{2} \mathrm{~d} y \mathrm{~d} \tau \\
& +C(\varepsilon, T) \int_{0}^{t} \int_{B_{1}(0)}|w(|y|, \tau)|^{2} \mathrm{~d} y \mathrm{~d} \tau .
\end{aligned}
$$


Substituting this estimate into (3.28) we obtain

$$
\begin{aligned}
\int_{B_{1}(0)}|w(|y|, t)|^{2} \mathrm{~d} y \leqslant & -\frac{1}{2} C_{1}(T) \int_{0}^{t} \int_{B_{1}(0)}|\nabla w(|y|, \tau)|^{2} \mathrm{~d} y \mathrm{~d} \tau \\
& +C(\varepsilon, T) \int_{0}^{t} \int_{B_{1}(0)}|w(|y|, \tau)|^{2} \mathrm{~d} y \mathrm{~d} \tau .
\end{aligned}
$$

By the Gronwall Lemma, this implies that

$$
\int_{B_{1}(0)}|w(|y|, t)|^{2} \mathrm{~d} y=0 \quad \text { for all } 0 \leqslant t \leqslant T .
$$

Hence $w \equiv 0$, or $v_{1} \equiv v_{2}$. This proves the uniqueness.

Finally, the assertion that $v_{0}^{\prime}(z) \geqslant 0$ implies $v_{\varepsilon z}(z, t) \geqslant 0$ follows from a similar argument to that in [9].

\section{The main result and its proof}

In this section we establish the main result. We first prove the following lemma:

LEMMA 4.1 Under the condition (2.19), for any $T>0$ the problem 2.11, 2.13, 2.15) and 2.18 has a solution $v$ on $[0,1] \times[0, T]$, satisfying: $v(|y|, t) \in W_{p}^{2,1}\left(Q_{T}\right)$ for any $1<p<\infty$, and

$$
0 \leqslant v(z, t) \leqslant \sigma_{S} \quad \text { for } 0 \leqslant z \leqslant 1,0 \leqslant t \leqslant T .
$$

If in addition $v_{0}^{\prime}(z) \geqslant 0$ then also $v_{z}(z, t) \geqslant 0$.

Proof. By Lemma 3.1. for every $\varepsilon>0$ the problem 3.3 -3.5 has a unique solution $v_{\varepsilon}=v_{\varepsilon}(z, t)$ on $[0,1] \times[0, T]$, and it satisfies 3.6 and 3.7). Take a fixed $p>5$. By (3.6) and the compact embedding

$$
W_{p}^{2,1}\left(Q_{T}\right) \hookrightarrow C^{\alpha, \alpha / 2}\left(\bar{Q}_{T}\right) \quad(0<\alpha<2-5 / p),
$$

it follows that we can find a sequence of positive numbers $\varepsilon_{k} \rightarrow 0$ (as $k \rightarrow \infty$ ) and a function $v \in W_{p}^{2,1}\left(Q_{T}\right)$ such that if we set $v_{k}=v_{\varepsilon_{k}}(k=1,2, \ldots)$ then

$$
\begin{gathered}
v_{k}(|y|, t) \rightarrow v(y, t), \quad \nabla v_{k}(|y|, t) \rightarrow \nabla v(y, t) \quad \text { uniformly for }(y, t) \in Q_{T}, \\
\nabla^{2} v_{k}(|y|, t) \rightarrow \nabla^{2} v(y, t), \quad v_{k t}(|y|, t) \rightarrow v_{t}(y, t) \quad \text { weakly in } L^{p}\left(Q_{T}\right) .
\end{gathered}
$$

Since every $v_{k}(|y|, t)$ is spherically symmetric in $y$, the limit $v(y, t)$ is also spherically symmetric in $y$, so that $v(y, t)=v(|y|, t)$. Furthermore, since for every $\varepsilon>0$,

$$
0 \leqslant H_{\varepsilon}\left(v_{\varepsilon}(|y|, t)-\sigma_{D}\right) \leqslant 1 \quad \text { for }(y, t) \in Q_{T},
$$

by replacing $\left\{\varepsilon_{k}\right\}$ with a suitable subsequence if necessary, we can further assume that

$$
H_{\varepsilon_{k}}\left(v_{k}(|y|, t)-\sigma_{D}\right) \rightarrow h(y, t) \quad * \text {-weakly in } L^{\infty}\left(Q_{T}\right),
$$

for some $h \in L^{\infty}\left(Q_{T}\right)$. Clearly, $h(y, t)$ is also spherically symmetric in $y$, so that later on we write $h=h(|y|, t)$. We assert that

$$
h(|y|, t)=\left\{\begin{array}{l}
1 \quad \text { for a.e. }(y, t) \in Q_{T} \text { such that } v(|y|, t)>\sigma_{D}, \\
0 \quad \text { for a.e. }(y, t) \in Q_{T} \text { such that } v(|y|, t)<\sigma_{D}
\end{array}\right.
$$


Indeed, by (4.2) and (4.3) it is easy to verify that for any $\delta>0, h(|y|, t)=1$ for a.e. $(y, t)$ in the set $\left\{(y, t) \in Q_{T}: v(|y|, t) \geqslant \sigma_{D}+\delta\right\}$. By the arbitrariness of $\delta$, we infer that $h(|y|, t)=1$ for a.e. $(y, t) \in Q_{T}$ such that $v(|y|, t)>\sigma_{D}$. Similarly we can prove that $h(|y|, t)=0$ for a.e. $(y, t) \in Q_{T}$ such that $v(|y|, t)<\sigma$. This proves the assertion. By (4.3) and 4.2) we also have

$$
F_{\varepsilon_{k}}\left(v_{k}, t\right) \rightarrow m(t) \quad * \text {-weakly in } L^{\infty}[0, T],
$$

where

$$
m(t)=\bar{\mu}\left\{\int_{0}^{1}(v(z, t)-\tilde{\sigma}) h(z, t) z^{2} \mathrm{~d} z-\bar{v} \int_{0}^{1}(1-h(z, t)) z^{2} \mathrm{~d} z\right\} .
$$

This further implies that

$$
\lim _{k \rightarrow \infty} \int_{0}^{t} F_{\varepsilon_{k}}\left(v_{k}, \tau\right) \mathrm{d} \tau=\int_{0}^{t} m(\tau) \mathrm{d} \tau \quad \text { for any } t \in[0, T]
$$

Taking $z=|y|$ and $\varepsilon=\varepsilon_{k}$ (correspondingly, replacing $v$ with $v_{k}$ ) in (3.3) and letting $k \rightarrow \infty$, we see that

$$
c v_{t}=R_{0}^{-2} \exp \left(-2 \int_{0}^{t} m(\tau) \mathrm{d} \tau\right) \Delta v+c m(t)(y \cdot \nabla v)-v h \text { on } Q_{T}
$$

in distribution sense, hence also a.e. on $Q_{T}$, because all terms in this equation are locally integrable functions. Since

$$
v_{t}=0, \quad \nabla v=0, \quad \Delta v=0 \quad \text { a.e. on the set }\left\{(y, t) \in Q_{T}: v(|y|, t)=\sigma_{D}\right\},
$$

by $(4.8)$ it follows that

$$
h=0 \quad \text { a.e. on the set }\left\{(y, t) \in Q_{T}: v(|y|, t)=\sigma_{D}\right\} .
$$

Hence

$$
h(|y|, t)=H\left(v(|y|, t)-\sigma_{D}\right) \quad \text { for a.e. }(y, t) \in Q_{T} .
$$

By (4.6) we further infer that

$$
m(t)=\bar{\mu}\left\{\int_{0}^{1}(v(z, t)-\tilde{\sigma}) H\left(v(z, t)-\sigma_{D}\right) z^{2} \mathrm{~d} z-\bar{v} \int_{0}^{1}\left(1-H\left(v(z, t)-\sigma_{D}\right)\right) z^{2} \mathrm{~d} z\right\}
$$

for a.e. $t \in[0, T]$. Substituting (4.10) into 4.8) we conclude that $v=v(z, t)$ is a solution of the equation (2.18). Since by the uniform convergence of $v_{k}$ and $\nabla v_{k}$ respectively to $v$ and $\nabla v$ it is clear that $v$ satisfies (2.11) and (2.13), we see that $v$ solves the problem 2.18, , 2.11) and (2.13). Finally, by taking the weak limit in (3.6) and 3.7) we immediately get the other assertions of the lemma.

By Lemma 4.1, we have the following main result of this paper:

THEOREM 4.2 Under the conditions $(1.7)$ and $(1.8)$, for any $T>0$ the problem 2.2 - 2.6 has a solution $(\sigma(r, t), R(t))$ for all $0 \leqslant t \leqslant T$, satisfying: (i) $R(t) \in C^{1-0}[0, T]$, and $R(t)>0$ for all $t \in[0, T]$; (ii) $\sigma(|x|, t) \in W_{p}^{2,1}\left(\tilde{Q}_{T}\right)$ for any $1<p<\infty$, where $\tilde{Q}_{T}=\left\{(x, t) \in \mathbb{R}^{3} \times \mathbb{R}:|x|<\right.$ $R(t), 0<t<T\}$, and

$$
0 \leqslant \sigma(r, t) \leqslant \sigma_{S} \quad \text { for } 0 \leqslant r \leqslant R(t), 0 \leqslant t \leqslant T .
$$

If in addition $\sigma_{0}^{\prime}(r) \geqslant 0$ then also $\sigma_{r}(r, t) \geqslant 0$. 


\section{Acknowledgments}

This work is supported by the National Natural Science Foundation of China under the grant number 10171112.

\section{REFERENCES}

1. AdAm, J. \& Bellomo, N. A Survey of Models for Tumor-Immune System Dynamics, Birkhäuser, Boston (1997). Zbl 0874.92020

2. BazAliy, B. \& Friedman, A. A free boundary problem for an elliptic-parabolic system: application to a model of tumor growth, Comm. Partial Differential Equations 28 (2003), 517-560. Zbl 1031.35151 MR 1976462

3. BAZAliy, B. \& FrIedman, A. Global existence and stability for an elliptic-parabolic free boundary problem: application to a model of tumor growth, Indiana Univ. Math. J. 52 (2003), 1265-1304. MR 2010327

4. Byrne, H. M. \& Chaplain, M. A. J. Growth of nonnecrotic tumors in the presence and absence of inhibitors, Math. Biosci. 130 (1995), 151-181. Zbl 0836.92011

5. Byrne, H. M. \& Chaplain, M. A. J. Growth of necrotic tumors in the presence and absence of inhibitors, Math. Biosci. 135 (1996), 187-216. Zbl 0856.92010

6. Byrne, H. M. \& Chaplain, M. A. J. Free boundary value problems associated with the growth and development of multicellular spheroids, European J. Appl. Math. 8 (1997), 639-658. Zbl 0906.92016 MR 1608619

7. CUI, S. Analysis of a mathematical model for the growth of tumors under the action of external inhibitors, J. Math. Biol. 44 (2002), 395-426. Zbl 1019.92017| MR 1908130

8. Cui, S. \& Friedman, A. Analysis of a mathematical model of the effect of inhibitors on the growth of tumors, Math. Biosci. 164 (2000), 103-137. Zbl 0998.92022 MR 1751267

9. Cui, S. \& Friedman, A. Analysis of a mathematical model of the growth of necrotic tumors, J. Math. Anal. Appl. 255 (2001), 636-677. Zbl 0984.35169 MR 1815805

10. CUI, S. \& FRIEDMAN, A. A hyperbolic free boundary problem modeling tumor growth, Interfaces Free Bound. 5 (2003), 159-181. Zbl 1040.35143 MR 1980470

11. Cui, S. \& Friedman, A. A free boundary problem for a singular system of differential equations: An application to a model of tumor growth, Trans. Amer. Math. Soc. 355 (2003), 3537-3590. Zbl 1036.34018 MR 1990162

12. Friedman, A. \& Reitich, F. Analysis of a mathematical model for the growth of tumors, J. Math. Biol. 38 (1999), 262-284. Zbl 0944.92018 MR 1684873

13. Friedman, A. \& Reitich, F. Symmetry-breaking bifurcation of analytic solutions to a free boundary problem: An application to a model of tumor growth, Trans. Amer. Math. Soc. 353 (2000), 1587-1634. Zbl 0983.35019 MR 1806728

14. Friedman, A. \& Reitich, F. On the existence of spatially patterned dormant malignancies in the model for the growth of non-necrotic vascular tumor, Math. Models Methods Appl. Sci. 11 (2001), 601-625. Zbl 1013.92024 MR 1832995

15. Greenspan, H. P. Models for the growth of a solid tumor by diffusion, Stud. Appl. Math. 52 (1972), 317-340. Zbl 0257.92001

16. Greenspan, H. P. On the growth and stability of cell cultures and solid tumors, J. Theor. Biol. 56 (1976), 229-242. MR 0429164

17. Jones, A. F., Byrne, H. M., Gibson, J. S., \& Dold, J. W. A mathematical model of the stress induced during avascular tumor growth, J. Math. Biol. 40 (2000), 473-499. Zbl 0964.92025 MR 1770937 
18. Pettet, G., Please, C. P., Tindall, M. J., \& McElwain, D. The migration of cells in multicell tumor spheroids, Bull. Math. Biol. 63 (2001), 231-257.

19. Sutherland, R. M. Cell and environmental interactions in tumor microregions: The multicell spheroid model, Science 240 (1988), 177-184.

20. WARD, J. P. \& KING, J. R. Mathematical modelling of avascular-tumour growth, IMA J. Math. Appl. Med. Biol. 14 (1997), 39-69. Zbl 0866.92011

21. WARD, J. P. \& KING, J. R. Mathematical modelling of avascular-tumour growth II: Modelling growth saturation, IMA J. Math. Appl. Med. Biol. 16 (1999), 171-211. Zbl 0943.92019 